\title{
Absorption of very-long-chain saturated fatty acids in totally hydrogenated fish oil
}

\author{
Linda Granlund ${ }^{1}$, Laila N. Larsen ${ }^{2}$, Erling N. Christiansen ${ }^{1}$ and Jan I. Pedersen ${ }^{1 *}$ \\ ${ }^{1}$ Institute for Nutrition Research and ${ }^{2}$ Institute of Medical Biochemistry, Faculty of Medicine, University of Oslo, 0316 Oslo, \\ Norway
}

(Received 12 January 2000 - Revised 20 June 2000 - Accepted 21 July 2000)

\begin{abstract}
Partially hydrogenated fish oil (PHFO) contains a high amount of trans fatty acids (TFA). Total hydrogenation results in a minimal amount of TFA, but a high content of very-long-chain saturated fatty acids (VLCSFA). Absorption and metabolism of VLCSFA from totally hydrogenated fish oil (THFO) were studied in rats. Groups of eight rats were fed one of four diets containing $40 \mathrm{~g}$ soyabean oil (SBO)/kg (low-fat diet), $150 \mathrm{~g} \mathrm{SBO} / \mathrm{kg}$ (SBO diet), $40 \mathrm{~g} \mathrm{SBO}+$ $110 \mathrm{~g}$ PHFO/kg (PHFO diet) or $40 \mathrm{~g} \mathrm{SBO}+110 \mathrm{~g}$ THFO $/ \mathrm{kg}$ (THFO diet) for 4 weeks. A lower absorption coefficient of the fat content was found in the THFO group (61\%) compared with the other groups (PHFO $95 \%$, SBO $99 \%$, low fat $98 \% ; P<0 \cdot 05$ ), which was mainly due to reduced absorption of VLCSFA. A reduced weight gain was found for the THFO group compared with the other groups, but this was only significant when compared with the SBO group $(P<0.05)$. Faecal fat excretion (dry weight) was markedly increased in the THFO group (47\%), which was $2 \cdot 4,4 \cdot 8$ and $8 \cdot 3$ times higher compared with the groups fed PHFO, SBO and low-fat diets $(P<0 \cdot 05)$, respectively. Serum total cholesterol was reduced for the PHFO and THFO groups $(P<0.05)$, whereas serum triacylglycerol was increased for the PHFO group compared with the other groups $(P<0 \cdot 05)$. Animals fed THFO diet had an increased content of 20:0 and 22:0 in the serum triacylglycerol fraction $(P<0.05)$, whereas only 20:0 was increased in the serum phospholipid fraction $(P<0.05)$. The low absorption coefficient of THFO must be considered if this fat is to be used for consumption by animals or man.
\end{abstract}

Dietary fat: Hydrogenated fish oil: Bioavailability

Appreciable quantities of fish oil are available for food production, but because of the stability, taste and flavour of this oil, processing, in practice partial hydrogenation, has been necessary before introducing it to, for example, margarine. Partial hydrogenation will, in addition to conversion of unsaturated fatty acids to saturated fatty acids, result in cis-trans isomerism, with formation of relatively high amounts of trans fatty acids (TFA). Intake of TFA is associated with increased risk of CHD (Ascherio et al. 1994). One alternative method for processing is total hydrogenation, which results in fat containing high amounts of long-chain saturated fatty acids (LCSFA) (stearic acid (18:0)) and very-long-chain saturated fatty acids (VLCSFA) (arachidic acid (20:0), behenic acid (22:0) and tetracosanoic acid (24:0)). Only minor amounts of TFA are formed during total hydrogenation of fish oil.

Kaplan \& Greenwood (1998) showed a low absorption of totally hydrogenated soyabean oil (SBO) compared with medium-chain triacylglycerols, hydrogenated coconut oil and SBO. They related the low bioavailability of totally hydrogenated SBO to the high content of LCSFA. Totally hydrogenated SBO is especially rich in stearic acid (18:0) (800 g/kg total fat content). High content of stearic acid (18:0) leads to an increased portion present as tristearin, which is shown to reduce stearic acid absorption (Mattson, 1959; Bergstedt et al. 1990). The relationship between stearic acid (18:0) and tristearin may also apply to fatty acids with longer chain length (Mattson, 1959), but not for mono- and polyunsaturated fatty acids with the same chain length (Bergstedt et al. 1990). No previous studies have been published regarding the absorption of VLCSFA from totally hydrogenated fish oil (THFO). Our hypothesis, based upon the results from Mattson (1959) and Kaplan \& Greenwood (1998), would be that reduced bioavailability

\footnotetext{
Abbreviations: LCSFA, long-chain saturated fatty acids; PHFO, partially hydrogenated fish oil; SBO, soyabean oil; TFA, trans fatty acids; THFO, totally hydrogenated fish oil; VLCSFA, very-long-chain saturated fatty acids.

* Corresponding author: Dr Jan I. Pedersen, fax +47 228513 41, email j.i.pedersen@basalmed.uio.no
} 
of THFO compared with partially hydrogenated fish oil (PHFO) is due to the increased content of VLCSFA. The purpose of the present study was to determine the absorption and metabolic effects of VLCSFA in THFO in comparison with SBO and PHFO.

\section{Methods \\ Animals and diets}

Male PVG rats (B\&K Universal AB, Sollentuna, Sweden), initially weighing about $120 \mathrm{~g}$, were fed one of four diets ad libitum for 4 weeks. The animals were housed individually in stainless-steel wire-bottomed cages specially designed for metabolic experiments (Hansen \& Holm, 1971), in a temperature- $\left(22-23^{\circ} \mathrm{C}\right)$ and humidity(40-60\%) controlled environment with a $12 \mathrm{~h}$ light-dark cycle. Diets were fed from food cups secured with a spring to minimize spillage. The local responsible veterinarian approved the protocol. The diet composition (Table 1) was as described by Thomassen et al. (1979), with small modifications. The diets contained $40 \mathrm{~g} \mathrm{SBO} / \mathrm{kg}$ (low-fat diet), $150 \mathrm{~g} \mathrm{SBO} / \mathrm{kg}$ (SBO diet), $40 \mathrm{~g} \mathrm{SBO}+110 \mathrm{~g} \mathrm{PHFO} /$ $\mathrm{kg}$ (PHFO diet) and $40 \mathrm{~g} \mathrm{SBO}+110 \mathrm{~g}$ THFO $/ \mathrm{kg}$ (THFO diet) respectively. The three high-fat diets contained $30 \%$ energy as fat, while the low-fat diet contained $9 \%$ energy from fat. The melting points of PHFO $\left(30-32^{\circ} \mathrm{C}\right)$ and THFO $\left(54-56^{\circ} \mathrm{C}\right)$ are markedly increased compared with the native oil, due to an increased content of saturated fatty acids after hydrogenation. For this reason PHFO and THFO were heated to $50^{\circ} \mathrm{C}$ and $65^{\circ} \mathrm{C}$ respectively, before blending the diet to an homogenous mixture. The fatty acid composition of the diets, determined by GC as described later, is shown in Table 2.

\section{Experimental design}

Animals were assigned to one of four dietary treatment groups ( $n 8$ per group) by controlled randomization such that mean body weight did not differ among the groups. All the animals consumed one of the four diets for 4 weeks.

Table 1. Composition of the four diets $(\mathrm{g} / \mathrm{kg})$

\begin{tabular}{lcc}
\hline & Low-fat diet & SBO, PHFO and THFO diets \\
\hline Cornstarch* & 480 & 370 \\
Casein $\dagger$ & 200 & 200 \\
Sucrose $\ddagger$ & 200 & 200 \\
Mineral mix $\dagger$ & 50 & 50 \\
Vitamin mix $\dagger$ & 15 & 15 \\
Cellulose§ & 10 & 10 \\
L-Methionine & 4 & 4 \\
SBOף & 40 & 40 \\
Additional oilף & 0 & 110 \\
\hline
\end{tabular}

SBO, soyabean oil; PHFO, partially hydrogenated fish oil, THFO, totally hydrogenated fish oil.

* Hydro Chemicals Norge A/S, Etterstad, Norway.

† ICN, Nerlien, Oslo, Norway.

¥ Obtained from a local store

$\S$ Norsk Medisinaldepot (drugstore)

II Sigma Aldrich, Oslo, Norway.

In SBO, PHFO (Margarit 30/32) or THFO (HMF 54/56) from Denofa AS

Fredrikstad, Norway. The source of the fish oil was a mix of several fish oils.
Table 2. Fatty acid composition $(\mathrm{g} / 100 \mathrm{~g})$ of the four diets fed during the experimental period*

\begin{tabular}{lrrrr}
\hline Fatty acid & Low fat & SBO & PHFO & THFO \\
\hline $14: 0$ & 0.19 & 0.11 & 7.53 & $4 \cdot 28$ \\
$15: 0$ & & & 0.54 & 0.60 \\
$16: 0$ & 12.41 & 12.20 & 23.31 & 23.44 \\
$16: 1 t$ & & & 6.11 & 0.24 \\
$16: 1$ & 0.10 & 0.10 & 2.92 & 0.19 \\
$17: 0$ & 0.09 & 0.09 & 0.42 & 0.76 \\
$17: 1 t$ & & & 0.07 & 0.15 \\
$17: 1$ & 0.04 & 0.04 & & \\
$18: 0$ & 3.58 & 3.51 & 6.03 & 20.49 \\
$18: 1 t$ & & & 5.86 & 0.28 \\
$18: 1$ & 23.85 & 23.95 & 14.01 & 9.22 \\
$19: 0$ & & & 0.27 & 0.35 \\
$18: 2 n-6$ & 53.09 & 53.31 & 18.97 & 20.2 \\
$20: 0$ & 0.27 & 0.27 & 1.56 & 8.09 \\
$20: 1 t$ & & & 4.17 & 0.51 \\
$20: 1+18: 3 n-3$ & 6.07 & 6.15 & 5.42 & 2.27 \\
$18: 4 n-6$ & 0.04 & 0.03 & 0.04 & \\
$21: 0$ & & & 0.10 & 0.26 \\
$22: 0$ & 0.26 & 0.27 & 1.03 & 7.48 \\
$22: 1 t$ & & & 2.36 & 0.20 \\
$22: 1$ & & & 1.87 & 0.08 \\
$24: 0$ & & & & 0.25 \\
\hline
\end{tabular}

SBO, soyabean oil; PHFO, partially hydrogenated fish oil, THFO, totally hydrogenated fish oil; $t$, trans.

* For details of composition of diets see Table 1.

Prior to the start of the experiment there was an adjustment period of $3 \mathrm{~d}$. Food intake and faecal excretion were measured daily, and the animals were weighed every $3 \mathrm{~d}$. The small amount of food wastage was weighed daily and subtracted. Diets and faeces were stored at $-20^{\circ} \mathrm{C}$. The animals were killed by $\mathrm{CO}_{2}$ exposure at the end of the experiment. The blood was withdrawn from the abdominal artery and the liver removed for isolation of peroxisomes.

\section{Diet analysis}

All diets were analysed for fat, protein (Kjeldahl analysis) and mineral (ash) content. The fat content was extracted using the method of Folch et al. (1957). Fatty acid analyses were performed as described later. To calculate the dietary energy available for absorption, fatty acids (the amount ingested and not excreted in faeces) were given the value of $39.4 \mathrm{~kJ} / \mathrm{g}$, which is the physical heat of combustion, whereas the Atwater value of $17 \mathrm{~kJ} / \mathrm{g}$ was used for proteins and carbohydrates.

\section{Faecal analysis}

The faecal excretion from each animal was mixed with a small portion of water, freeze-dried, ground with a mortar and pestle into an homogenous mixture and stored at $-20^{\circ} \mathrm{C}$. Lipid was extracted using a modification of the method of Folch et al. (1957). A small amount of water $(0.25 \mathrm{ml})$ was added to $0.25 \mathrm{~g}$ freeze-dried faeces. Methanol $(2.5 \mathrm{ml})$ and chloroform $(5 \mathrm{ml})$ were added to each tube, which were shaken and heated at $60^{\circ} \mathrm{C}$ for $1 \mathrm{~h}$ (Kamei et al. 1995). The samples were centrifuged, reheated and transferred to a second test tube. The extraction was performed twice. $\mathrm{KCl}$ in water $(8 \cdot 8 \mathrm{~g} / \mathrm{l})$ 
was added to each sample prior to centrifugation. The lipid phase was dried under $\mathrm{N}_{2}$ and put in an exicator to obtain complete dryness (Kaplan \& Greenwood, 1998). Faeces from the group fed THFO contained an interphase of nonextracted fat after the first extraction. This interface was saponified by addition of $\mathrm{KOH}$ in methanol $(2 \mathrm{ml}, 150 \mathrm{~g} / \mathrm{l})$ and heated at $65^{\circ} \mathrm{C}$ for $45 \mathrm{~min}$. $\mathrm{HCl}$ was added to $\mathrm{pH} 1-2$, and the sample was extracted three times with hexane ( $3 \mathrm{ml}$ ). The fatty acid extract was dried under $\mathrm{N}_{2}$ and put in an exicator for complete drying. Heating was necessary to complete the extraction in both procedures because of the increased melting point of PHFO and THFO compared with the native oil. The faeces samples from each diet group were further analysed for protein (Kjeldahl analysis) and mineral (ash) content.

\section{Serum analysis}

The concentration of triacylglycerol and total cholesterol in serum was measured by enzyme kits obtained from Bio Mérieux (Marcy l'Etoile, France). Total lipids were extracted by the method of Folch et al. (1957). Phospholipids and triacylglycerols were separated by TLC (hexane-diethylether-acetic acid (80:20:1, by vol.)).

\section{Fatty acid analysis}

The extracted fatty acids from faeces and diets were methylated with benzene and methanolic HCL (3 M) (Supelco Inc., Bellefonte, PA, USA) using the method of Hoshi et al. (1973). Tricosanoic acid (23:0) was added as an internal fatty acid standard (corresponding to approximately $10 \%$ fatty acids content). Fatty acids from the serum phospholipid and triacylglycerol fractions were transmethylated (methanolic $\mathrm{HCl}(3 \mathrm{M})$, benzene, dimethoxypropane) using the method of Hoshi et al. (1973). L- $\alpha$-Phosphatidylcholine diheptadecanoyl and triheptadacanic acid were used as internal standard for phospholipids and triacylglycerols respectively. The fatty acid composition of diets, faeces, phospholipid and triacylglycerol fractions were analysed by GC (Shimadzu GC-17A; Shimadzu, Kyoti, Japan) with a polar Supelco capillary column (SP-2560, $100 \mathrm{~m} \times 0.25 \mathrm{~mm}$; Supelco Inc.) and flame ionization detection. The carrier gas was He. Retention times were verified with purified standards (Nu Chek Prep Inc., Elysian, MN, USA).

\section{Other assays}

Peroxisomes were isolated from liver tissue by subcellular fractionation (Prydz et al. 1988) and density-gradient centrifugation (Ruyter et al. 1992). The peroxisomal fatty acid oxidation activity was determined by measuring the activity of the rate-limiting enzyme fatty acid oxidase, using a method developed by Small et al. (1985) with minor modifications (Spydevold \& Bremer, 1989).

\section{Statistical analyses}

A one-way ANOVA Bonferroni test for variance was used to compare the group variances, except where data from only two groups were available. In these cases Student's $t$ test was used. For all analyses, the acceptable level of significance (type 1 error) was $P \leq 0 \cdot 05$. Statistical

Table 3. Apparent fatty acid absorption ( $\mathrm{mmol}$ ) in rats fed on one of four diets for 4 weeks§ (Mean values and standard deviations for eight rats per group)

\begin{tabular}{|c|c|c|c|c|c|c|c|c|}
\hline \multirow[b]{2}{*}{ Fatty acid } & \multicolumn{2}{|c|}{ Low fat } & \multicolumn{2}{|c|}{ SBO } & \multicolumn{2}{|c|}{ PHFO } & \multicolumn{2}{|c|}{ THFO } \\
\hline & Mean & SD & Mean & SD & Mean & SD & Mean & $\mathrm{SD}$ \\
\hline $14: 0$ & 0.10 & 0.01 & 0.23 & 0.02 & $16 \cdot 60^{*} \dagger$ & 0.68 & $14.90^{*} † \ddagger$ & 0.73 \\
\hline $15: 0$ & & & & & $1 \cdot 10$ & 0.11 & $1.73 \ddagger$ & 0.10 \\
\hline $16: 0$ & $7 \cdot 20$ & 0.56 & $26 \cdot 90^{*}$ & $1 \cdot 14$ & $49 \cdot 10^{*} \dagger$ & $2 \cdot 80$ & $50 \cdot 90^{*} \dagger$ & 3.73 \\
\hline $16: 1 t$ & & & & & $13 \cdot 40$ & 0.60 & $0.62 \ddagger$ & 0.03 \\
\hline $16: 1$ & 0.04 & 0.01 & 0.20 & 0.01 & $6 \cdot 60^{*} \dagger$ & 0.26 & $0.45^{\star} † \ddagger$ & 0.03 \\
\hline $17: 0$ & 0.03 & 0.01 & 0.17 & 0.01 & $0.83^{*} \dagger$ & 0.07 & $1 \cdot 25^{\star} \dagger \ddagger$ & 0.13 \\
\hline $17: 1$ & 0.01 & 0.00 & $0.06^{*}$ & 0.01 & $0 \cdot 15^{\star} \dagger$ & 0.01 & $0 \cdot 30^{*} \dagger \ddagger$ & 0.04 \\
\hline $18: 0$ & 2.00 & 0.18 & $7 \cdot 30^{*}$ & 0.40 & $11.60^{*} \dagger$ & $1 \cdot 10$ & $26 \cdot 90^{*} \dagger \ddagger$ & 3.30 \\
\hline $18: 1 t$ & & & & & $12 \cdot 00$ & $1 \cdot 20$ & & \\
\hline $18: 1$ & $14 \cdot 30$ & $1 \cdot 10$ & $54 \cdot 10^{*}$ & $2 \cdot 50$ & $31 \cdot 10^{*} \dagger$ & 1.40 & $21.00^{*} \dagger \ddagger$ & $2 \cdot 30$ \\
\hline 19:0 & & & & & 0.50 & 0.03 & $0.18 \ddagger$ & 0.04 \\
\hline $18: 2 n-6$ & 31.90 & $2 \cdot 40$ & $120 \cdot 60^{*}$ & $5 \cdot 60$ & $42 \cdot 90^{*} \dagger$ & $1 \cdot 70$ & $47 \cdot 90^{*} \dagger$ & 2.96 \\
\hline $20: 0$ & 0.13 & 0.02 & 0.50 & 0.06 & $2 \cdot 90^{*} \dagger$ & 0.30 & $5 \cdot 64^{*} \ddagger$ & $1 \cdot 13$ \\
\hline $20: 1 t$ & & & & & 7.90 & 0.90 & $0.17 \ddagger$ & 0.03 \\
\hline $20: 1+18: 3 n-3$ & 3.60 & 0.30 & $13.90^{*}$ & 0.64 & $11 \cdot 70^{\star} \dagger$ & 0.66 & $5 \cdot 60^{*} \pitchfork \ddagger$ & 0.34 \\
\hline $18: 4 n-6$ & 0.01 & 0.01 & $0.06^{*}$ & 0.01 & $0.09^{*} \dagger$ & 0.00 & & \\
\hline $21: 0$ & & & & & 0.13 & 0.04 & 0.09 & 0.04 \\
\hline $22: 0$ & 0.12 & 0.02 & 0.49 & 0.07 & $2 \cdot 40^{*} \dagger$ & 0.09 & $3 \cdot 62^{*} † \ddagger$ & 1.07 \\
\hline $22: 1 t$ & & & & & $4 \cdot 20$ & 0.68 & $0.10 \ddagger$ & 0.09 \\
\hline $22: 1$ & & & & & 3.58 & 0.43 & & \\
\hline Total & 59.40 & 0.42 & 234.50 & 0.87 & $218 \cdot 80$ & 0.70 & 181.40 & 0.95 \\
\hline
\end{tabular}

SBO, soyabean oil; PHFO, partially hydrogenated fish oil; THFO, totally hydrogenated fish oil; $t$, trans.

Mean values were significantly different from the low-fat group: ${ }^{\star} P<0.05$; mean values were significantly different from the SBO group: $† P<0.05$; mean values were significantly different from the PHFO group: $\ddagger P<0.05$.

$\S$ Ingested fatty acids that are not excreted in the faeces (mmol fatty acid apparently absorbed during the whole feeding period). For details of composition of diets see Tables 1 and 2 . 
Table 4. Absorption coefficients of fatty acids (\%) in rats fed one of four diets for 4 weeks $\S$

(Mean values and standard deviations for eight rats per group)

\begin{tabular}{|c|c|c|c|c|c|c|c|c|}
\hline \multirow[b]{2}{*}{ Fatty acid } & \multicolumn{2}{|c|}{ Low fat } & \multicolumn{2}{|c|}{ SBO } & \multicolumn{2}{|c|}{ PHFO } & \multicolumn{2}{|c|}{ THFO } \\
\hline & Mean & SD & Mean & SD & Mean & SD & Mean & SD \\
\hline $14: 0$ & 84 & 6 & 90 & 4 & $98^{*} \dagger$ & 1 & $87 \ddagger$ & 4 \\
\hline $15: 0$ & & & & & 88 & 7 & $74 \ddagger$ & 5 \\
\hline $16: 0$ & 97 & 2 & 98 & 2 & 93 & 4 & $62^{*} \dagger \ddagger$ & 5 \\
\hline $16: 1 t$ & & & & & 97 & 2 & $74 \ddagger$ & 5 \\
\hline $16: 1$ & 61 & 14 & $90^{*}$ & 5 & $99^{*}$ & 1 & $88^{*}$ & 5 \\
\hline $17: 0$ & 56 & 22 & $84^{*}$ & 9 & $88^{*}$ & 6 & 47†‡ & 5 \\
\hline $17: 1$ & 43 & 28 & $82^{*}$ & 8 & $87^{\star}$ & 5 & $62 \ddagger$ & 9 \\
\hline $18: 0$ & 92 & 5 & 92 & 5 & 84 & 7 & $39^{*} \dagger \ddagger$ & 5 \\
\hline $18: 1 t$ & & & & & 87 & 7 & & \\
\hline $18: 1$ & 98 & 1 & 99 & 0 & $96 \dagger$ & 3 & $92^{*} \dagger \ddagger$ & 1 \\
\hline 19:0 & & & & & 90 & 4 & $21 \ddagger$ & 4 \\
\hline $18: 2 n-6$ & 99 & 0 & 99 & 0 & $99^{*} \dagger$ & 1 & $99^{\star} \dagger \ddagger$ & 0 \\
\hline $20: 0$ & 80 & 12 & 85 & 8 & 81 & 7 & $23^{*} \dagger \ddagger$ & 4 \\
\hline $20: 1 t$ & & & & & 82 & 8 & $53 \ddagger$ & 11 \\
\hline $20: 1+18: 3 n-3$ & 96 & 3 & 97 & 2 & $84^{*} \dagger$ & 7 & $85^{\star} \dagger$ & 2 \\
\hline $18: 4 n-6$ & 48 & 29 & $78^{*}$ & 12 & $99^{*}$ & 1 & & \\
\hline $21: 0$ & & & & & 61 & 16 & $13 \ddagger$ & 5 \\
\hline $22: 0$ & 79 & 12 & 82 & 8 & $97^{*} \dagger$ & 1 & $18^{\star} \dagger \ddagger$ & 5 \\
\hline $22: 1 t$ & & & & & 71 & 11 & $21 \neq$ & 19 \\
\hline $22: 1$ & & & & & 78 & 8 & & \\
\hline Total & 98 & & 99 & & 95 & & 61 & \\
\hline
\end{tabular}

SBO, soyabean oil; PHFO, partially hydrogenated fish oil; THFO, totally hydrogenated fish oil; $t$, trans.

Mean values were significantly different from the low-fat group: ${ }^{\star} P<0.05$; mean values were significantly different from the SBO group: $† P<0.05$; mean values were significantly different from the PHFO group: $\ddagger P<0.05$.

$\S$ Ingested fatty acids that are not excreted in the faeces $\%$ fatty acids apparently absorbed during the feeding period). For details of the composition of diets see Tables 1 and 2 .

analysis was conducted using SPSS 9.0 (SPSS Inc., Chicago, IL, USA).

\section{Results}

\section{Apparent lipid absorption}

The apparent lipid absorption calculated as the difference between fatty acid content in diet and faeces (Monsma $e t$ al. 1996; Kaplan \& Greenwood, 1998), expressed as mmol as well as percentage absorption (absorption coefficient) are shown in Tables 3 and 4 respectively. For the fatty acids in the THFO diet the absorption coefficient was markedly reduced $(61 \%)$ compared with the other groups (low fat $98 \%$, SBO $99 \%$, PHFO $95 \%$; $P<0.05$ ); however, in terms of amount (mmol), the absorption of LCSFA and VLCSFA (18:0, 20:0 and 22:0) was higher compared with the other groups. The absorption coefficient for the different saturated fatty acids decreased with increasing chain length, a tendency which was most pronounced in the group fed THFO diet. The absorption of VLCSFA was also lower compared with unsaturated fatty acids with the same chain length. In addition to reduced absorption of VLCSFA, there was a reduced absorption of TFA (16:1 trans; 18:1 trans, 20:1 trans and 22:1 trans) in the group fed THFO diet compared with the PHFO diet $(P<0 \cdot 05)$. The absorption coefficient of PHFO and THFO may be underestimated due to the supplementation of these diets with SBO to provide a sufficient amount of essential fatty acids.

The difference in absorption coefficients between the four diets results in different energy densities of the diets. The energy available for absorption, assuming that the three high-fat diets were isoenergetic, has been calculated, as well as the energy actually absorbed using the lipid absorption coefficients (Table 5). The calculations of energy actually absorbed were performed taking the differences in protein excretion into consideration (Table 6), but assuming no difference in carbohydrate absorption. The dietary energy intake available for absorption did not differ between the four groups. However, the energy actually absorbed differed among the groups, with the animals fed THFO diet absorbing the lowest amount. The absorbed energy in the THFO group was lower compared with the SBO and PHFO groups $(P<0 \cdot 05)$, but also compared with the low-fat group, although this difference was not significant. The slightly increased food intake did not appear to compensate for the reduced amount of available energy in the diet containing THFO.

Table 5. Experimental variables related to growth and metabolism for rats fed one of the four diets for 4 weeks§ (Mean values and standard deviations for eight rats per group)

\begin{tabular}{|c|c|c|c|c|c|c|c|c|}
\hline \multirow[b]{2}{*}{ Variable } & \multicolumn{2}{|c|}{ Low fat } & \multicolumn{2}{|c|}{ SBO } & \multicolumn{2}{|c|}{ PHFO } & \multicolumn{2}{|c|}{ THFO } \\
\hline & Mean & SD & Mean & SD & Mean & SD & Mean & $\overline{S D}$ \\
\hline Energy available for absorption ( $\mathrm{kJ} / 4$ weeks)\| & 8010 & 689 & 8170 & 499 & 8288 & 472 & 8401 & 540 \\
\hline Apparent energy absorbed (kJ/4 weeks) & 7252 & 642 & 7520 & 463 & 7509 & 431 & $6780 \dagger \ddagger$ & 441 \\
\hline Body wt gain $(\mathrm{g} / 28 \mathrm{~d})$ & 111.4 & 18.5 & 121.4 & $15 \cdot 2$ & 118.0 & $9 \cdot 0$ & $98.0 \dagger$ & $15 \cdot 2$ \\
\hline Total food intake ( $\mathrm{g} / 28 \mathrm{~d})$ & $441 \cdot 1$ & 38.7 & $398 \cdot 4^{*}$ & $24 \cdot \overline{3}$ & $404 \cdot 1$ & 23.0 & $409 \cdot 6$ & $26 \cdot 3$ \\
\hline Relative liver wt ( $\mathrm{g} / \mathrm{kg}$ body weight) & 46 & 46 & 46 & 3 & 48 & 2 & 46 & 2 \\
\hline Total serum cholesterol (mM) & 3.9 & 0.6 & 4.0 & 0.3 & $3.3+$ & 0.3 & $3 \cdot 3^{*} \dagger$ & 0.5 \\
\hline Serum triacylglycerol (mM) & $2 \cdot 2 \ddagger$ & 0.5 & $2.5 \ddagger$ & 0.7 & 4.5 & 1.0 & $2.0 \ddagger$ & 0.3 \\
\hline Fatty acid oxidase ${ }^{\star \star}$ & $134 \cdot 3$ & $44 \cdot 2$ & $196 \cdot 7^{*}$ & $72 \cdot 9$ & $212 \cdot 2^{*}$ & 79.2 & $139 \cdot 6 \ddagger$ & 21.2 \\
\hline
\end{tabular}

SBO, soyabean oil; PHFO, partially hydrogenated fish oil; THFO, totally hydrogenated fish oil.

Mean values were significantly different from the low-fat group: ${ }^{*} P<0.05$; mean values were significantly different from the SBO group: $\dagger P<0.05$; mean values were significantly different from the PHFO group: $\ddagger P<0.05$.

$\S$ For details of composition of diets see Tables 1 and 2.

|| Calculation based on the assumption that all high-fat diets provided $20508 \mathrm{~kJ} / \mathrm{kg}$ and the low-fat diet $18066 \mathrm{~kJ} / \mathrm{kg}$.

ๆ Apparent energy absorbed calculated by using adjusted energy density based on the apparent lipid absorption coefficient and protein absorption measured as protein intake in the diet minus faecal excretion.

** Expressed as $\mathrm{nmol}$ dichlorofluorecin oxidized/min per mg protein. 
Table 6. Faecal contents of rats fed one of the four diets for 4 weeks§

(Mean values and standard deviations for eight rats per group)

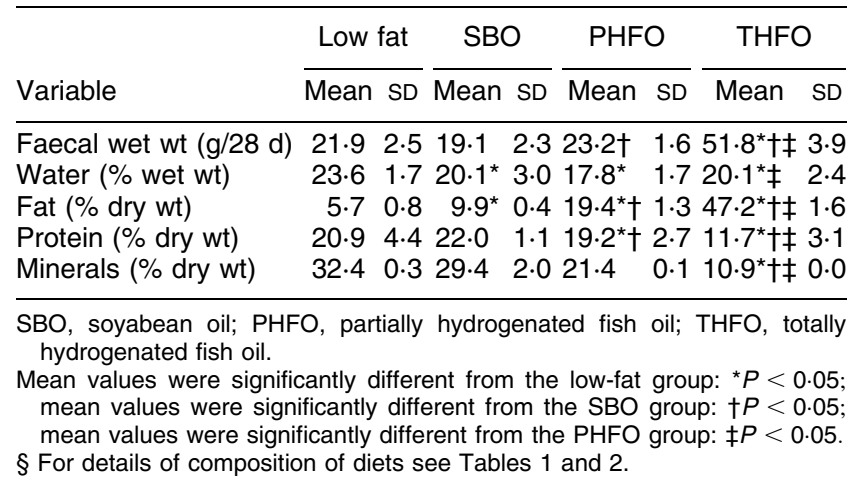

\section{Variables related to growth and metabolism}

A summary of the body-weight gain, food intake, relative liver weight, total serum cholesterol, serum triacylglycerol and liver fatty acid oxidase activity is shown in Table 5. Animals fed the THFO diet had a lower weight gain compared with the group fed SBO diet $(P<0.05)$. Total food intake during the experimental period was higher for the group fed the low-fat diet compared with the animals fed SBO diet $(P<0 \cdot 05)$. However, there was no significant difference in food intake between the THFO group and the other groups. The relative liver weights did not differ among the four dietary groups. Animals fed PHFO and THFO diets had lower serum total cholesterol concentration compared with the group fed SBO diet $(P<$ $0 \cdot 05)$, and concentrations in the THFO group were also lower compared with the low-fat group $(P<0 \cdot 05)$. The animals fed the PHFO diet had increased serum triacylglycerol concentrations compared with the other groups $(P<$ $0 \cdot 05)$. Fatty acid oxidase activity was increased in the groups fed SBO and PHFO diets compared with the low-fat group $(P<0.05)$, and was also increased in the PHFO group compared with the THFO group $(P<0.05)$.

\section{Faeces analysis}

Faecal excretion (wet weight) as well as percentage of fat, protein and minerals in faeces is shown in Table 6. Faecal excretion (wet weight) was markedly increased for the THFO group compared with the other groups $(P<0.05)$ and also increased in the PHFO group compared with the SBO group $(P<0 \cdot 05)$. Faeces excreted by rats fed THFO diet contained a higher percentage of fat $(47 \%)$ than the faeces excreted by the PHFO fed animals (19\%), which in turn, contained a higher percentage than that from the animals fed SBO $(10 \%)$ and low-fat $(6 \%)$ diets $(P<$ $0 \cdot 05)$. The percentage protein was decreased for the PHFO and THFO groups compared with the other groups $(P<$ $0.05)$, in addition to the THFO group having reduced protein content compared with the PHFO group $(P<$ $0 \cdot 05)$. The percentage faecal mineral content was reduced in the THFO group compared with all the other groups $(P<0 \cdot 05)$.
Table 7. Fatty acid composition $(\mathrm{g} / 100 \mathrm{~g})$ of the serum triacylglycerol fraction in rats fed one of four diets for 4 weeks§

(Mean values and standard deviations for eight rats per group)

\begin{tabular}{|c|c|c|c|c|c|c|c|c|}
\hline \multirow[b]{2}{*}{ Fatty acid } & \multicolumn{2}{|c|}{ Low fat } & \multicolumn{2}{|c|}{ SBO } & \multicolumn{2}{|c|}{ PHFO } & \multicolumn{2}{|c|}{ THFO } \\
\hline & Mean & SD & Mean & SD & Mean & SD & Mean & $\mathrm{s}$ \\
\hline $4: 0$ & 3 & 0.5 & $1 \cdot 1$ & 1 & $5 \cdot 8^{*} \dagger$ & 0.4 & $\cdot 2^{*} \dagger$ & \\
\hline 14: & $0 \cdot 1$ & 0.0 & & & 0.1 & J & & \\
\hline $15: 0$ & 0.3 & 0.1 & 0.3 & 0.3 & 0.6 & & $0.7^{*} \dagger$ & \\
\hline $6: 0$ & $32 \cdot 4$ & 2.9 & $22 \cdot 2^{\star}$ & $5 \cdot 0$ & $26 \cdot 0^{*}$ & 1.5 & $28.8 \dagger$ & \\
\hline 6: & 0.7 & 0.1 & 0.4 & 0.3 & $6 \cdot 4^{*} \dagger$ & 0.4 & $1.0 \dagger \ddagger$ & 0 . \\
\hline $6: 1$ & $6 \cdot 1$ & $1 \cdot 8$ & $1 \cdot 1^{*}$ & 0.5 & $3 \cdot 1^{*} \dagger$ & 0.4 & $2 \cdot 2^{*}$ & \\
\hline 17: & $0 \cdot 1$ & 0.0 & $2 \cdot 0$ & 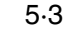 & 0.2 & 0 & 0.2 & \\
\hline 7: & 0.1 & 0.1 & $0 \cdot 1$ & $0 \cdot 1$ & 0. & & 0.1 & \\
\hline 18. & $2 \cdot 4$ & 0.3 & $6 \cdot 6$ & $8 \cdot 3$ & $3 \cdot 4$ & 3 & $8 \cdot 4$ & \\
\hline 18: & & & & & $6 \cdot 3$ & 0.3 & $0.5 \ddagger$ & \\
\hline 18:1 & 31.0 & $4 \cdot 8$ & $24 \cdot 3$ & $7 \cdot 1$ & $19 \cdot 8^{*}$ & 0.9 & $22 \cdot 4^{*}$ & \\
\hline 9.0 & & & & & 0. & 0.2 & & \\
\hline $8: 2$ & $20 \cdot 3$ & $4 \cdot 0$ & 33.1 & $13 \cdot 5$ & $14.8 \dagger$ & 1.9 & 23.9 & \\
\hline $20:$ & 0.1 & 0.0 & 0.5 & 0 & 0 & 0.1 & $1.5^{\star} \dagger \ddagger$ & \\
\hline 18:3 & $0 \cdot 1$ & 0.0 & 0.1 & 0. & $0 \cdot 1^{*} \dagger$ & 0.0 & $0 \cdot 1$ & \\
\hline & & & & & $2 \cdot 2$ & 0.2 & & \\
\hline $20: 2 n-6,9 t t$ & & & & & $1 \cdot 0$ & 0.2 & & \\
\hline 0 & 1.9 & 0.7 & $2 \cdot 1$ & 0.9 & ${ }^{*} \dagger$ & 0.4 & $2 \cdot 1 \ddagger$ & \\
\hline 20: & $0 \cdot 1$ & 0.1 & 0.3 & 0 & $0.1 \dagger$ & 0.0 & $0.1 \dagger$ & \\
\hline $20: 4 n$ & 1.5 & 1.4 & $4 \cdot 2$ & 8. & 0.4 & 0.1 & 1.0 & \\
\hline 21: & 0.1 & 0.0 & $0 \cdot 1$ & 0.1 & $0 \cdot 2^{*} \dagger$ & 0.1 & $0.1 \ddagger$ & \\
\hline 22:0 & 0.1 & 0.0 & 0.4 & 0.7 & 0.3 & 0.1 & $1 \cdot 0^{*} \dagger \ddagger$ & \\
\hline 2.11 & & & & & 1. & 0.2 & & \\
\hline $22: 2 t t+22: 2$ & & & & & 0.2 & 0.1 & & \\
\hline $22: 1$ & & & & & $1 \cdot 1$ & 0.2 & & \\
\hline $22: 4 n-$ & 0.3 & 0.2 & 0.3 & 0.2 & $0 \cdot 1^{*} \dagger$ & 0.0 & 0.2 & \\
\hline $22: 6 n$ & 0.3 & 0.2 & 0.6 & 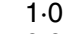 & 0 . & 0.0 & 0.3 & \\
\hline $24: 0$ & $0 \cdot 1$ & 0.0 & $0.1^{*} \ddagger$ & 0.0 & $0 \cdot 1$ & 0.0 & $0.1 \dagger$ & 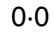 \\
\hline
\end{tabular}

SBO, soyabean oil; PHFO, partially hydrogenated fish oil; THFO, totally hydrogenated fish oil; $t$, trans; $c$, cis.

Mean values were significantly different from the low-fat group: ${ }^{\star} P<0.05$; mean values were significantly different from the SBO group: $\dagger P<0.05$; mean values were significantly different from PHFO group: $\ddagger P<0.05$.

$\S$ For details of composition of diets see Tables 1 and 2.

\section{Fatty acids in serum triacylglycerol and phospholipid fractions}

The percentage arachidic (20:0) and behenic acid (22:0) in the serum triacylglycerol fraction was higher in the group fed THFO diet compared with the other groups $(P<0.05)$ (Table 7). However, in the phospholipid fraction only arachidic acid was increased for the PHFO and THFO groups compared with the low-fat group $(P<0.05)$ (Table 8).

\section{Discussion}

This present study shows a low absorption coefficient of fat in the THFO diet compared with the other diets. This observation is in agreement with earlier studies on totally hydrogenated SBO and VLCSFA (Jandacek et al. 1993; Kaplan \& Greenwood, 1998). The absorption coefficient indicates that nearly $40 \%$ fat in the THFO diet was not absorbed, which is in agreement with the study of Kaplan \& Greenwood (1998), who showed a bioavailability coefficient of totally hydrogenated SBO of $41.5 \%$. In their study, totally hydrogenated SBO contributed $40 \%$ total energy content in the diet, compared with $30 \%$ energy as fat in our study. 
Table 8. Fatty acid composition $(\mathrm{g} / 100 \mathrm{~g})$ of the serum phospholipid fraction in rats fed one of four diets for 4 weeks§

(Mean values and standard deviations for eight rats per group)

\begin{tabular}{|c|c|c|c|c|c|c|c|c|}
\hline \multirow[b]{2}{*}{ Fatty acid } & \multicolumn{2}{|c|}{ Low fat } & \multicolumn{2}{|c|}{ SBO } & \multicolumn{2}{|c|}{ PHFO } & \multicolumn{2}{|c|}{ THFO } \\
\hline & Mean & $\overline{S D}$ & Mean & $\overline{S D}$ & Mean & $\overline{S D}$ & Mean & $\mathrm{SD}$ \\
\hline $14: 0$ & 0.6 & 0.2 & 0.7 & 0.8 & $1 \cdot 1$ & 0.6 & 0.9 & 0.6 \\
\hline $15: 0$ & 0.2 & 0.0 & 0.2 & 0.2 & 0.3 & 0.1 & $0.4^{*} \dagger$ & 0.1 \\
\hline $16: 0$ & 27.8 & 3.6 & $26 \cdot 5$ & 3.8 & $22 \cdot 6^{\star}$ & 3.0 & $25 \cdot 4$ & 3.8 \\
\hline $16: 1 t$ & 0.3 & 0.1 & 0.3 & 0.1 & $3 \cdot 1^{*} \dagger$ & $1 \cdot 3$ & $0.3 \ddagger$ & 0.1 \\
\hline $16: 1$ & 1.1 & 0.3 & $0.4^{*}$ & 0.2 & 0.8 & 0.5 & $0.5^{\star}$ & 0.4 \\
\hline $17: 1 t$ & 0.3 & 0.1 & 0.3 & 0.1 & 0.3 & 0.1 & 0.3 & 0.0 \\
\hline $17: 1$ & 0.1 & 0.0 & 0.1 & 0.0 & 0.1 & 0.1 & 0.1 & 0.0 \\
\hline 18:0 & 21.5 & $7 \cdot 8$ & $26 \cdot 0$ & $3 \cdot 3$ & $18.5 \dagger$ & $2 \cdot 2$ & $25 \cdot 8 \ddagger$ & 1.4 \\
\hline $18: 1 t$ & & & & & $6 \cdot 2$ & 0.9 & $0.3 \ddagger$ & 0.2 \\
\hline $18: 1$ & 11.4 & $6 \cdot 2$ & $8 \cdot 2$ & $4 \cdot 2$ & $11 \cdot 2$ & $2 \cdot 6$ & 5.9 & 1.6 \\
\hline 19:0 & & & & & 0.3 & 0.1 & & \\
\hline $18: 2 n-6$ & $19 \cdot 7$ & $8 \cdot 6$ & $20 \cdot 3$ & $4 \cdot 1$ & 21.5 & $4 \cdot 2$ & $24 \cdot 2$ & 2.9 \\
\hline $20: 0$ & 0.1 & 0.0 & 0.2 & 0.2 & $0.3^{*}$ & 0.1 & $0.3^{*}$ & 0.1 \\
\hline $18: 3 n-3$ & 0.1 & 0.1 & $0 \cdot 1$ & 0.0 & 0.1 & 0.1 & $0 \cdot 1$ & 0.0 \\
\hline $20: 1 t$ & & & & & 0.4 & 0.3 & & \\
\hline $20: 2 n-6,9 t t$ & & & & & 0.1 & 0.2 & & \\
\hline $20: 1$ & 0.7 & 0.6 & 0.5 & 0.7 & $1 \cdot 2$ & 1.5 & 0.3 & 0.4 \\
\hline $20: 2 n-6$ & 0.2 & 0.1 & $0.4^{*} \ddagger$ & 0.2 & 0.2 & 0.1 & $0.2 \dagger$ & 0.1 \\
\hline $20: 4 n-6$ & $13 \cdot 1$ & $6 \cdot 1$ & $12 \cdot 6$ & 6.9 & $8 \cdot 3$ & $5 \cdot 3$ & $12 \cdot 1$ & $3 \cdot 1$ \\
\hline $21: 0$ & & & & & 0.1 & 0.1 & & \\
\hline $22: 0$ & 0.2 & 0.1 & 0.1 & 0.2 & 0.1 & 0.0 & 0.2 & 0.1 \\
\hline $22: 1 t$ & & & & & 0.1 & 0.1 & & \\
\hline $22: 1$ & & & & & 0.2 & 0.1 & & \\
\hline $22: 4 n-6$ & 0.2 & 0.1 & 0.1 & 0.1 & 0.1 & 0.1 & $0 \cdot 1$ & 0.0 \\
\hline $22: 6 n-3$ & 1.9 & 1.0 & 1.9 & 1.2 & 1.8 & 1.3 & 1.6 & 0.6 \\
\hline $24: 0$ & 0.1 & 0.0 & $0.1^{*} \dagger$ & 0.0 & 0.1 & 0.0 & $0.1 \dagger$ & 0.0 \\
\hline
\end{tabular}

SBO, soyabean oil; PHFO, partially hydrogenated fish oil; THFO, totally hydrogenated fish oil; $t$, trans; $c$, cis.

Mean values were significantly different from the low-fat group: ${ }^{\star} P<0.05$; mean values were significantly different from the SBO group: $\dagger P<0.05$; mean values were significantly different from the PHFO group: $\ddagger P<0.05$. $\S$ For details of composition of diets see Tables 1 and 2.

The low bioavailability of THFO was mainly due to low absorption of LCSFA and VLCSFA, which decreased with increasing chain length of the fatty acids. In the study by Kaplan \& Greenwood (1998), the low absorption of totally hydrogenated SBO was related to the high content of saturated fatty acids, especially stearic acid (18:0) that amounted to $70 \%$. Furthermore, Apgar et al. (1987) showed low absorption of cocoa butter, which was also due to a high content of saturated fatty acids. The content of stearic acid (18:0) in cocoa butter is $300-360 \mathrm{~g} / \mathrm{kg}$ (Gunstone et al. 1986). In our study the THFO diet contained $200 \mathrm{~g}$ stearic acid/kg (18:0), which is much higher compared with the other diets (low-fat and SBO $40 \mathrm{~g} / \mathrm{kg}$, PHFO $60 \mathrm{~g} / \mathrm{kg}$ ). Consequently, a high content of tristearin in the THFO diet could be the reason for low absorption of stearic acid (18:0) in this group (39\%) compared with the other groups (84-95\%). The mechanism responsible for reduced absorption of stearic acid (18:0) as tristearin could be reduced or slow lipolysis of triacylglycerol containing stearic acid (18:0), as indicated by Bergstedt et al. (1990). Increased content of nonadecanoic (19:0) and heneicosanoic acid (21:0), as well as pentadecanoic (15:0) and palmitic acid (16:0) in the diet also resulted in reduced absorption of these fatty acids, as was indicated by Mattson (1959). Jandacek et al. (1993) showed lower absorption of triacylglycerol with increasing chain length of the saturated fatty acids. They found behenic acid (22:0) to have absorption of 19-29\%, which is in agreement with our present study of an absorption of $18 \%$ in the group fed THFO. The absorption of arachidic (20:0) and tetracosanoic acid (24:0) was 36-41 \% and 2$25 \%$ in Jandacek's study (Jandacek et al. 1993), which is higher compared with our study, where the absorption was $23 \%$ for arachidic acid (20:0) and no absorption for tetracosanoic acid (24:0).

Very-long-chain polyunsaturated fatty acids are most often found in position 2 in fish oil triacylglycerol (Sebedio, 1992). Due to hydrogenation, and since pancreatic lipase hydrolyses mainly in $s n-1$ and $s n-3$ positions, this will result in VLCSFA 2-monoacylglycerols. It is to be expected that this will contribute to the reduced absorption of saturated fatty acids in the PHFO and THFO groups.

Peters et al. (1997) showed that Olestra, which is a fat substitute, affects the absorption of other compounds, depending on the lipophilic character of the compounds. This could also be a reason for the reduced absorption of saturated fatty acids with shorter chain length in the THFO diet.

In addition to reduced absorption of saturated fatty acids in the THFO group, the absorption of TFA was reduced compared with fatty acids in cis configuration. This difference in absorption of isomeric fatty acids was mainly seen in the group fed THFO, but also in the group fed PHFO. The dietary content of TFA was markedly higher in the PHFO diet compared with the THFO diet, which indicates that the mechanism responsible for the reduced absorption of TFA is different from that of saturated fatty acids. Cis-trans configuration affects the polarity of a compound, with cis configuration being the more polar. TFA with higher lipophilic character can result in increased aggregation with non-absorptive saturated fatty acids (Peters et al. 1997), which can lead, furthermore, to reduced absorption compared with fatty acids in cis configuration.

The reduced absorption coefficient of THFO did not result in increased food intake, which is in contrast to the results of Kaplan \& Greenwood (1998). A lower content of fat in our study (30\% energy) compared to Kaplan \& Greenwood's study (40 \% energy), in addition to higher absorption coefficient for THFO (61\%) compared with totally hydrogenated SBO $(42 \%)$, could be a reason for this disagreement.

The weight gain can be considered in relation to the available energy content in the diet. The THFO group had lower weight gain compared with the other groups, which may be associated with the reduced content of available energy of this diet.

Our present study showed decreased serum total cholesterol concentration in the PHFO and THFO groups compared with the other groups, which is in agreement with Duthie et al. (1988) who showed a lower concentration in rats fed PHFO compared with partially hydrogenated SBO and rapeseed oil. It should be noted that the distribution of HDL- and LDL-cholesterol in rats and man is very different, with rats having the major part of cholesterol present as HDL-cholesterol (Weisgraber et al. 1977). In our present study, the reduction in the PHFO 
group could be due to the high amount of TFA which reduces HDL-cholesterol (Mensink \& Katan, 1989), while in the THFO group it could be due to reduced absorption of cholesterol (Chen et al. 1989; Jandacek et al. 1993). The PHFO and THFO diets contain higher amounts of stearic (18:0) and behenic acid (22:0), as well as other very-longchain saturated and monounsaturated fatty acids, which could contribute to the reduced concentration of serum total cholesterol in these groups (Hill et al. 1990; Jandacek et al. 1993; Kritchevsky, 1994).

The increased serum triacylglycerol concentration after feeding PHFO found in our present study is not in agreement with other studies in rats (Duthie et al. 1988) or human subjects (Almendingen et al. 1995). The reason for this disagreement is not known.

Incorporation of fatty acids into serum triacylglycerol and phospholipid can be used as a marker for the absorption of fatty acids from the lumen and utilization of the fatty acids in the organism respectively. The difference in incorporation of VLCSFA in serum triacylglycerol (20:0 and 22:0) and phospholipid (20:0) can be due to a selective incorporation of these fatty acids into the phospholipid fraction compared with the triacylglycerol fraction (Larsen et al. 1998).

Ishii et al. (1980) and Neat et al. (1981) showed an induction of the peroxisomal $\beta$-oxidation after intake of high-fat diets, which is in agreement with our present study with increased fatty acid oxidase activity in liver peroxisomes of animals fed SBO and PHFO diets compared with the other groups. SBO, PHFO and THFO diets are all highfat diets, with a fat content of $30 \%$ energy and the increase in peroxisomal activity for the SBO and PHFO groups can be explained by the high-fat content available from these diets. Induction of peroxisomal fatty acid oxidase activity after intake of PHFO has also been shown in other studies (Christiansen et al. 1979; Neat et al. 1980; Flatmark et al. 1988), and has been associated with a high content of TFA (Christiansen et al. 1979). The THFO diet cannot be regarded as a high-fat diet due to the low bioavailable fat content.

To summarize, we found reduced absorption of VLCSFA and TFA from the THFO diet compared with unsaturated fatty acids and fatty acids with cis configuration. Both serum total cholesterol and triacylglycerol concentrations were reduced for the THFO group compared with the PHFO group, although this was only significant for the triacylglycerol concentration. Except for the high faecal excretion of fat, we did not observe any harmful effects of VLCSFA during the study period. However, before THFO can be used for consumption by man there is a need for more studies on the effects of these fatty acids in human subjects.

\section{Acknowledgements}

The authors wish to thank Halvor Holm, Aud Jørgensen and Hans J. Grav for valuable advice. Denofa AS and The Association of Norwegian Margarine Producers supported this study.

\section{References}

Almendingen K, Jordal O, Kierulf P, Sanstad B \& Pedersen JI (1995) Effects of partially hydrogenated fish oil, partially hydrogenated soybean oil and butter on serum lipoproteins and Lp (a) in men. Journal of Lipid Research 36, 1370-1384.

Apgar JL, Shively CA \& Tarka SMJ (1987) Digestibility of cocoa butter and corn oil and their influence on fatty acid distribution in rats. Journal of Nutrition 117, 660-665.

Ascherio A, Hennekens CH, Buring JU, Master C, Stampfer MJ \& Willet WC (1994) Trans-fatty acids intake and risk of myocardial infarction. Circulation 89, 94-101.

Bergstedt SE, Hayashi H, Kritchevsky D \& Tso P (1990) A comparison of absorption of glycerol tristearate and glycerol trioleate by rat small intestine. American Journal of Physiology 259, G386-G393.

Chen IS, Subramaniam S, Vahouny GV, Cassidy MM, Ikeda I \& Kritchevsky D (1989) A comparison of the digestion and absorption of cocoa butter and palm kernel oil and their effects on cholesterol absorption in rats. Journal of Nutrition 119, 1569-1573.

Christiansen RZ, Christiansen EN \& Bremer J (1979) The stimulation of erucate metabolism in isolated rat hepatocytes by rapeseed oil and marine oil-containing diets. Biochimica et Biophysica Acta 573, 417-429.

Duthie F, Barlow SM, Ashby R, Tesh JM, Whitney JC, Saunders A, Chapman E, Norum KR, Svaar H \& Opstvedt J (1988) Feeding of partially hydrogenated fish oil to rats in comparison with partially hydrogenated soybean oil and refined rapeseed oil. Acta Medica Scandinavica Supplementum 726, 189.

Flatmark T, Nilsson A, Kvannes J, Eikhom TS, Fukami MH, Kryvi H \& Christiansen EN (1988) On the mechanism of induction of the enzyme systems for peroxisomal $\beta$-oxidation of fatty acids in rat liver by diets rich in partially hydrogenated fish oil. Biochimica et Biophysica Acta 962, 122-130.

Folch J, Lees M \& Stanley GHS (1957) A simple method for the isolation and purification of total lipides from animal tissues. Journal of Biological Chemistry 226, 497-509.

Gunstone FD, Harwood JL \& Padley FB (1986) The Lipid Handbook, pp. 59 and 452 London, New York: Chapman and Hall.

Hansen L \& Holm H (1971) Apparatus for collecting faeces and urine from rats in metabolic experiments. Laboratory Animals 5, 221-224.

Hill JO, Peters JC, Swift LL, Yang D, Sharp T, Abumrad N \& Greene HL (1990) Changes in blood lipids during six days of overfeeding with medium or long chain triglycerides. Journal of Lipid Research 31, 407-416.

Hoshi M, Williams M \& Kishimoto Y (1973) Esterification of fatty acids at room temperature by chloroform-methanolic $\mathrm{HCl}-$ cupric acetate. Journal of Lipid Research 14, 599-601.

Ishii H, Fukumori N, Horie S \& Suga T (1980) Effects of fat content in the diet on hepatic peroxisomes of the rat. Biochimica et Biophysica Acta 617, 1-11.

Jandacek RJ, Hollenbach EJ, Kuehlthau CM \& Steimle AR (1993) Effects of dietary behenate and a caprenin-like fat on lipids in the hamster. Journal of Nutritional Biochemistry 4, 243-249.

Kamei M, Ohgaki S, Kanbe T, Niiya I, Mizutani H, MatsuiYuasa I, Otani S \& Morita S (1995) Effects of highly hydrogenated soybean oil and cholesterol on plasma, liver cholesterol and fecal steroids in rats. Lipids 30, 533-539.

Kaplan JR \& Greenwood CE (1998) Poor digestibility of fully hydrogenated soybean oil in rats: A potential benefit of hydrogenated fats and oils. Journal of Nutrition 128, 875-880.

Kritchevsky D (1994) Stearic acid metabolism and atherogenesis: 
history. American Journal of Clinical Nutrition 60, 997S1001S.

Larsen LN, Bremer J, Flock S \& Skattebøl L (1998) $\alpha$ - and $\beta$ alkyl-substituted eicosapentaenoic acids. Incorporation into phospholipids and effects on prostaglandin $\mathrm{H}$ synthase and 5lipoxygenase. Biochemical Pharmacology 55, 405-411.

Mattson FH (1959) The absorbability of stearic acid when fed as a simple or mixed triglyceride. Journal of Nutrition 69, 338-342.

Mensink RP \& Katan MB (1989) Effect of diet enriched with monounsaturated or polyunsaturated fatty acids on levels of low-density and high-density lipoprotein cholesterol in healthy women and men. New England Journal of Medicine 321, 436441.

Monsma CC, Gallaher DD \& Ney DM (1996) Reduced digestibility of beef tallow and cocoa butter affects bile acid excretion and reduces hepatic esterified cholesterol in rats. Journal of Nutrition 126, 2028-2035.

Neat CE, Thomassen MS \& Osmundsen H (1980) Induction of peroxisomal $\beta$-oxidation in rat by high-fat diets. Biochemical Journal 186, 369-371.

Neat CE, Thomassen MS \& Osmundsen H (1981) Effects of highfat diets on hepatic fatty acid oxidation in the rat. Biochemical Journal 196, 149-159.

Peters JC, Lawson KD, Middleton SJ \& Triebwasser KC (1997) Assessment of nutritional effects of olestra, a nonabsorbed fat replacement: introduction and overview. Journal of Nutrition 127, 1546S-1593S.

Prydz K, Kase BF, Bjørkhem I \& Pedersen JI (1988) Subcellular localization of $3 \alpha, 7 \alpha, 12 \alpha$-trihydroxy-5 $\beta$-cholestanoyl-coenzyme A ligase(s) in rat liver. Journal of Lipid Research 29, 997-1004.

Ruyter B, Lund JS, Thomassen MS \& Christiansen EN (1992) Studies of dihydroxyacetone phosphate acyltransferase in rat small intestine. Subcellular localization and effect of partially hydrogenated fish oil and clofibrate. Biochemical Journal 282, $565-570$.

Sebedio JL (1992) Oils \& Fats Manual. A Comprehensive Treatise. Properties, Production, Applications, vol. 1, pp. 266-276 [A Karleskind, editor]. London, Paris, New York: Intercept Ltd.

Small GM, Burdett K \& Connock MJ (1985) A sensitive spectrophotometric assay for peroxisomal acyl-CoA oxidase. Biochemical Journal 227, 205-210.

Spydevold O \& Bremer J (1989) Induction of peroxisomal $\beta$ oxidation in $7800 \mathrm{C} 1$ Morris hepatoma cells in steady state by fatty acids and fatty acid analogues. Biochimica et Biophysica Acta 1003, 72-79.

Thomassen MS, Strøm E, Christiansen EN \& Norum KR (1979) Effect of marine oil and rapeseed oil on consumption of fatty acids in lipoprotein triacylglycerides from rat blood plasma and liver perfusate. Lipids 14, 58-65.

Weisgraber KH, Mahley RW \& Assmann G (1977) The rat arginine-rich apoprotein and its redistribution following injection of iodinated lipoproteins into normal and hypercholesterolemic rats. Atherosclerosis 28, 121-140. 